Journal of Animal and Veterinary Advances 11 (3): 393-399, 2012

ISSN: $1680-5593$

(C) Medwell Journals, 2012

\title{
cDNA Cloning, Sequence Identification and Tissue Expression Profile of a Novel Gene FAR1 From Banna Mini-Pig Inbred Line (BMI)
}

\author{
${ }^{1,2}$ Pei Wang, ${ }^{1-3}$ Jinlong Huo, ${ }^{3}$ Heng Xiao and ${ }^{1,2}$ Yangzhi Zeng \\ ${ }^{1}$ Key Laboratory of Banna Mini-Pig Inbred Line of Yunnan Province, \\ 650201 Kunming, Yunnan, P.R. China \\ ${ }^{2}$ Faculty of Animal Science and Technology, \\ Yunnan Agricultural University, 650201 Kunming, Yunnan, P.R. China \\ ${ }^{3}$ Faculty of Life Science, Yunnan University, \\ 650091 Kunming, Yunnan, P.R. China
}

\begin{abstract}
The complete CDS sequence of Banna Mini-pig Inbred line (BMI) gene FAR1 was amplified using the Reverse Transcriptase-polymerase Chain Reaction (RT-PCR) based on the conserved sequence information of the cattle or other mammals and known highly homologous swine ESTs. This novel gene was then deposited into NCBI database and assigned to Accession No.: JF944893. Sequence analysis revealed that the BMI FAR1 encodes a protein of 515 amino acids that has high homology with the fatty acyl-CoA reductase 1 proteins of seven species-cattle $98 \%$, horse $98 \%$, mouse $98 \%$, orangutan $97 \%$, human $97 \%$, monkey $97 \%$ and rat $92 \%$. The phylogenetic tree analysis revealed BMI FAR1 has a closer genetic relationship with the bovine, human, orangutan and monkey FAR1 than with those of horse, mouse and rat. Analysis by RT-PCR showed that BMI FARl gene was over-expressed in muscle, lung, ovary, skin, large intestine, spleen, small intestine and nerve fiber and almost not expressed in other 10 tissues. Several microRNA target sites were predicted in the CDS of BMI FAR1 mRNA for further studying this gene in the future. The 3D structure of the FAR1 by homology modeling was similar to that of pseudomonas 3-alpha-hydroxysteroid dehydrogenase ( $2 \mathrm{dkn}$ Chain: A). The experiment will establish a foundation for further insight into this swine gene.
\end{abstract}

Key words: Banna Mini-pig Inbred line (BMI), pig, FAR1, tissue expression analysis, homology modeling, China

\section{INTRODUCTION}

Long-Chain Fatty Alcohol molecules (LCFAs) serve essential biological roles in a vast majority of living organisms (Gilbert and Chino, 1974; Gilby, 1980; Blomquist et al., 1987; Stanley and Nelson, 1993). Fatty acyl-CoA reductase is a key enzyme in LCFAs metabolism which catalyzes the NADPH-dependent reduction of fatty acyl-CoA precursors into fatty alcohols on the cytosolic side of the peroxisomal membrane in a two-step reaction that does not release intermediate aldehyde forms (Wanders and Waterham, 2006). Subsequently, fatty alcohols either incorporation into ether lipids or into waxes (Cheng and Russell, 2004). Waxes (oxygen esters of fatty alcohols and fatty acids) are major components of biological systems and fill a variety of functions. They are found on the surfaces of plants, insects and mammals and provide protection against various stresses such as desiccation, wetting and pathogen attack and are produced commercially at levels approaching 3 billion pounds per year for use in polishes, cosmetics and packaging (Cheng and Russell, 2004; Metz et al., 2000).
Ether lipids account for $\sim 20 \%$ of phospholipids in the human body and are precursors for platelet activating factor for cannabinoid receptor ligands and for essential membrane components in cells of the reproductive and nervous systems (Nagan and Zoeller, 2001; Munn et al., 2003).

There are two isoforms of mammalian FAR. FAR1, prefers saturated and unsaturated fatty acyl-CoAs of 16-18 carbon atoms as substrates and expressed in many tissues with the highest level in the preputial gland, a modified sebaceous gland; FAR2 is reactive with saturated acyl-CoAs of 16 or 18 carbon atoms only and mainly expressed in the eyelids which contains wax-laden meibomian glands. Both FAR1 and FAR2 expression was observed in the brain, a tissue rich in ether lipids. These findings suggest that fatty alcohol synthesis in mammals is accomplished by two FAR enzymes (FAR1 and FAR2) and expressed at high levels in tissues known to synthesize wax esters and ether lipids (Cheng and Russell, 2004; Wanders and Waterham, 2006). In support of a role for FAR that goes beyond LCFAs synthesis, positive regulation of its activity has been reported in association

Corresponding Author: Yangzhi Zeng, Key Laboratory of Banna Mini-Pig Inbred Line of Yunnan Province, 650201 Kunming, Yunnan, P.R. China 
with many important biological metabolism. For example, in Arabidopsis thaliana, FAR proteins have been identified as the Male Sterility 2 (MS2) gene product which is implicated in male gametogenesis. Mutations in MS2 inhibit the synthesis of exine (sporopollenin), rendering plants unable to reduce pollen wall fatty acids to corresponding alcohols (Chen et al., 2011). Moreover, FAR involves biosynthesis of a wide range of aliphatic fatty alcohols which are chemicals of the honey bee communication pheromones and is responsible for the production of sex pheromone bombykol in silkmoth (Bombyx mori) (Moto et al., 2003; Teerawanichpan et al., 2010).

Based on before described about the FARl gene, it is necessary to isolate this gene from pig for it is associated with energy metabolism, health and other important biological functions of animals. But until today the porcine FAR1 has not been reported yet. Surprisingly, the Banna Mini-pig Inbred line (BMI) was exploited by Yunnan Agricultural University from 1980s based on the small-ear pigs at Xishuangbanna, Yunnan province. A pair of progenitors was a sow and her son. Then, the propagation was conducted by means of highly full sibling or parent-offspring inbreeding and each generation underwent the strict selection.

As heterozygotic genes were separated and recombined in the process of inbreeding, BMI has already owned six families and eighteen substrains with different phenotypes and genotypes. Due to their consistent genetic background and minor interindividual differences, $\mathrm{BMI}$ is considered as an ideal model organism for biological studies (Crabbe et al., 2005; Yu et al., 2004; Zeng and Zeng, 2005).

The objective of this study was to isolate the full length coding sequence of BMI FARl gene according to the conserved sequence information of cattle or other mammals and highly homologous swine ESTs sequence information, conduct sequence analysis and some necessary function analysis of established nucleotide sequence, finally examine the expression in a range of BMI tissues. These will provide a primary foundation for further research on this porcine gene.

\section{MATERIALS AND METHODS}

Samples collection, RNA extraction and first-strand cDNA synthesis: Three matured female BMI were slaughtered for sampling. Fresh tissues (lymph node, midbrain, ovary, diencephalon, cerebrum, liver, kidney, spleen, heart, lung, nerve fiber, stomach, small intestine, large intestine, pancreas, skin, muscle and fat) were instantly frozen in liquid nitrogen and stored at $-80^{\circ} \mathrm{C}$ before use. Total RNA was extracted using the RNAiso Plus (TaKaRa, Dalian) according to the manufacturer's instructions. To remove genomic DNA contamination, total RNA was digested with RNase-free DNase I (TaKaRa, Dalian). Three micrograms of RNA were reverse transcribed with oligo $(\mathrm{dT})_{18}$ primer and M-MLV reverse transcriptase (Invitrogen, USA). The efficiency of reverse transcription was checked on $2 \%$ agarose gels stained with ethidium bromide.

Isolation of the BMI FAR1 gene: The GenBank FAR1 sequences for human (Accession No. NM 032228), cattle (Accession No. NM_001099032) and their highly homologous pig ESTs sequences: AJ662192, BI186799, DV938225, EV905565 and EW578845 were used to design a primer pair to amplify the complete coding sequence of FAR1 by using Primer Premier 5.0 software. The primers for BMI FARl gene were: 5'TGCGTCTAGGATCAGAATGG $-3^{\prime}$ and $5^{\prime}-$ GTCTTCAGTATCTCATAGTGCTG -3'. RT-PCR was performed to isolate the BMI FAR1 using the pooled cDNAs from different tissues above. The $25 \mu \mathrm{L}$ reaction system was $2.0 \mu \mathrm{L}$ cDNA (25 ng $\left.\mu \mathrm{L}^{-1}\right), 2.0 \mu \mathrm{L} 2.5 \mathrm{mM}$ mixed dNTPs (TaKaRa, Dalian), $2.5 \mu \mathrm{L} 10 \times$ Taq DNA polymerase buffer $\left(\mathrm{Mg}^{2+}\right.$ Plus $), 0.5 \mu \mathrm{L} 10 \mu \mathrm{M}$ forward primer, $0.5 \mu \mathrm{L} 10 \mu \mathrm{M}$ reverse primer, $0.25 \mu \mathrm{L}$ Taq DNA polymerase ( $5 \mathrm{U}_{\mu} \mathrm{L}^{-1}$, TaKaRa, Dalian) and $17.25 \mu \mathrm{L}$ sterile water. The PCR program initially started with $94^{\circ} \mathrm{C}$ denaturation for $2 \mathrm{~min}$ followed by 35 cycles of $94^{\circ} \mathrm{C} / 30 \mathrm{sec}, 55^{\circ} \mathrm{C} / 40 \mathrm{sec}, 72^{\circ} \mathrm{C} / 1.5 \mathrm{~min}$ then $72^{\circ} \mathrm{C}$ extension for $10 \mathrm{~min}$, finally $4^{\circ} \mathrm{C}$ to terminate the reaction. After the PCR the gene product was cloned into pMD18-T vector (TaKaRa, Dalian) and sequenced bidirectionally with the commercial fluorometric method. At least five independent clones were sequenced.

Bioinformatics analysis: Sequence analysis of BMI $F A R 1$ gene was performed using software in NCBI (http://www.ncbi.nlm.nih.gov) and ExPaSy (http://www.expasy.org). The cDNA sequence were predicted using the GenScan software (http://genes.mit. edu/GENSCAN. html). The Blastp program and Conserved Domain Architecture Retrieval tool were used to search for similar proteins and conserved domain, respectively (http://www.ncbi.nlm.nih.gov/Blast). The alignment of the nucleotide sequences and deduced amino acid sequences were computed using ClusterX and the phylogenetic trees were computed using the ClustalX and Mega 4.0 softwares with standard parameters. The theoretical isoelectric point $(\mathrm{pI})$ and Molecular weight $(\mathrm{Mw})$ were predicted using the compute $\mathrm{pI} / \mathrm{Mw}$ tool (http://us.expasy.org/tools/pi_tool.html). The putative signal peptide was predicted using the SignalP 3.0 server (http:/www.cbs.dtu.dk/services/SignalP/). The putative protein subcellular localization was predicted using PSort II (http://psort.hgc.jp/). Transmembrane topology prediction was performed using TMHMM-2.0 server (http://www.cbs.dtu.dk/services/TMHMM-2.0/). 
Secondary structures of deduced amino acid sequences were predicted with SOPMA (http://npsapbil.ibcp.fr/). The 3D structures was predicted based on the existed 3D structures by the amino acids homology modeling on swiss server (http://swissmodel. expasy.org/). Web-based microRNA (miRNA) predicting programs were used to locate conserved potential miRNA targets (http://www.mirbase.org/).

Semi-quantitative RT-PCR: To characterize the FAR1 gene further, RT-PCR was conducted to determine its expression in $18 \mathrm{BMI}$ tissues. To eliminate the effect of cDNA concentration, the researchers repeated the RTPCR five times using 1-5 $\mu \mathrm{L}$ cDNA as templates. The researchers selected the housekeeping gene $18 \mathrm{~S} r R N A$ (NR_002170) as a positive control.

The control primers used were 5'- GGACATCTAAG GGCATCACAG -3' and 5'- AATTCCGATAACGAACG AGACT -3'. The BMI FAR1 primers which were used to perform the semi-quantitative RT-PCR for tissue expression profile analysis were the same as the primers for isolation RT-PCR above. The PCR reactions were optimized for a number of cycles to ensure product intensity within the linear phase of amplification.

\section{RESULTS AND DISCUSSION}

Isolation and identification of BMI $F A R 1$ cDNA: Through RT-PCR with pooled tissue cDNAs for BMI FARl gene, the resulting PCR products was 1568 bp
(Fig. 1). This cDNA nucleotide sequence analysis using the BLAST software at NCBI server revealed that BMI FAR1 gene was not homologous to any of the known porcine genes and it was then deposited into the GenBank database (Accession No.: JF944893).

The sequence prediction was carried out using the GenScan software and results showed that the $1568 \mathrm{bp}$ cDNA sequence represent a single gene which encoded 515 amino acids.

The complete CDS and the encoded amino acids were shown in Fig. 2. The theoretical isoelectric point (pI) and Molecular weight $(\mathrm{Mw})$ were computed using

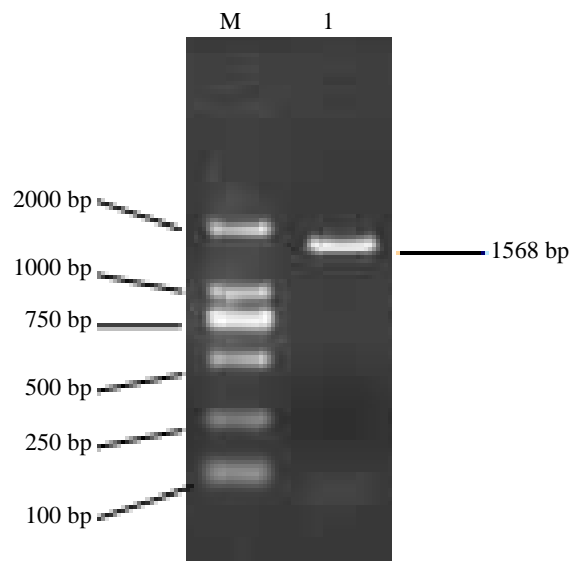

Fig. 1: RT-PCR result for BMI FAR1 gene; M: DL2000 DNA marker, 1: PCR product

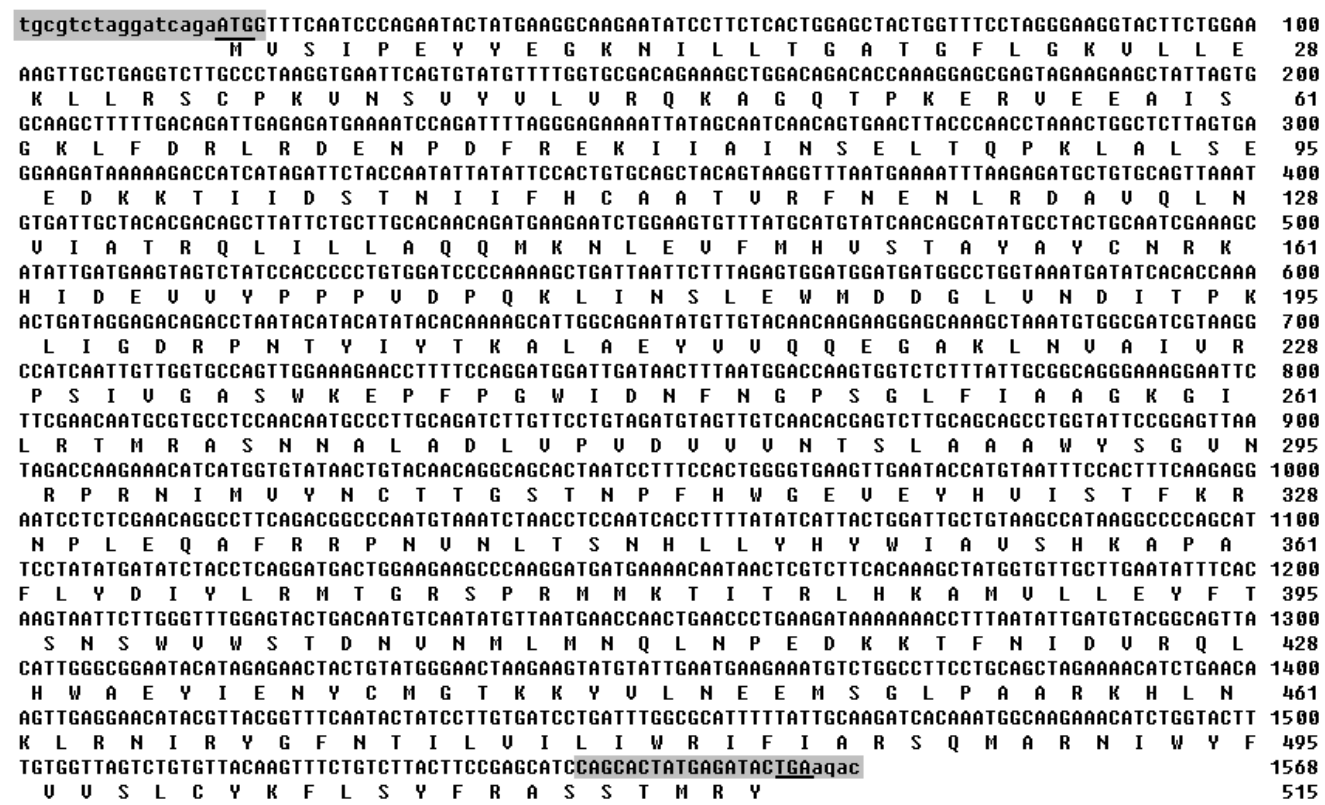

Fig. 2: The complete cDNA sequence and amino acid sequence of the protein encoded by FAR1 (GenBank Accession No.: JF944893). ATG, start codon; TGA, stop codon; gray highlighted nucleotide sequence, primers 
the compute $\mathrm{pI} / \mathrm{Mw}$ tool. The $\mathrm{pI}$ and the molecular weight of BMI FAR1 are 9.38 and 59273.66, respectively.

Functional prediction of BMI FAR1: The result from SignalP revealed that there is has no putative signal peptide in BMI FAR1 and indicated that it was probably a non-secretory protein. The potential protein subcellular localization prediction by Reinhardt's Method showed that BMI FAR1 was probably located in the cytoplasm with up to $89 \%$ probability. The cytoplasmic targeting localization of the human and mouse FAR1 protein had been confirmed (Cheng and Russell, 2004). Transmembrane topology prediction indicated that one transmembrane sequence (466-483AA) of BMI FAR1 was found with high probability. Proteins often contained several domains, each of which had their own evolutionary origins and functions. Examined using the Conserved Domain Architecture Retrieval tool of blast at the NCBI server (http://www.ncbi.nlm.nih.gov/BLAST) indicated that BMI FAR1 contains two separated conserved domains-FAR-N_SDR_e and FAR_C (from 11-330 amino acid residues and from 355-446 amino acid residues, respectively). The putative protein was also analyzed using prosite (http://expasy.org/prosite/) and SMART (http://smart.embl-heidelberg.de/) software.

Five kinds sites were found which were Casein kinase II phosphorylation sites (3-SipE-6, 51-TpkE-54, 94-SeeD97, 100-TiiD-103, 235-SwkE-238); Protein kinase C phosphorylation sites (51-TpK-53, 61-SgK-63, 114-TvR-116,193-TpK-195, 235-SwK-237,264-TmR-266, 325-TfK-327, 356-ShK-358, 371-TgR-373, 374-SpR-376, 441-TkK-443, 512-TmR-514), Tyrosine kinase phosphorylation site (160-RkhiDevvY-168), Nmyristoylation sites (219-GaklNV-224, 252-GLfiAA-257, 260-GIlrTM-265, 452-GLpaAR-457) and N-glycosylation site (283-NTSL-286, 304-NCTT-307, 341-NLTS-344) (Fig. 3). The prediction of secondary structure by SOPMA indicates that the deduced BMI FAR1 consists of alpha helices (266 AA), extended strands (71 AA), beta turn (20 AA) and random coils (158 AA) (Fig. 4). In order to better understand the detailed structure of BMI FAR1, the homology modeling of FAR1 was performed to estimate its 3D structure based on the existed 3D structure of the pseudomonas 3-alpha-hydroxysteroid dehydrogenase (2dkn Chain: A) (Fig. 5). From 3D structure, alpha helices can be seen obviously which represent a transmembrane domain with high probability. The 3D structure analysis may provide a basis for further studying the relationship between structure and function of FAR1.

MicroRNAs are noncoding single-stranded RNA molecules of 17-24 nucleotides that can regulate gene expression by binding to the coding region of target mRNAs (Bartel, 2004; Zeng et al., 2003). The researchers use web-based microRNA (miRNA) predicting programs to locate conserved potential miRNA targets: miRBase (http://www.mirbase.org/). The results showed four Sus scrofa microRNAs (ssc-miR-542-3p, ssc-miR-22-5p, ssc-miR-542-3p and ssc-miR-361-3p) were found to have the predicted target sites (209-uuugacagauugagagaugaaa230, 195-uuaguggcaagcuuu-209, 741-gauggauugauaacuu uaa-759 and 1438-ugugauccugauung-1452) in the BMI FAR1 sequence, respectively.

Further BLAST analysis revealed that the BMI FAR1 protein has high homology with the Fatty Acyl-CoA Reductase 1 (FAR1) proteins of seven species-cattle $(98 \%)$, horse $(98 \%)$, mouse $(98 \%)$, orangutan $(97 \%)$, human (97\%), monkey (97\%) and rat (92\%) (Fig. 6). Based on the alignment result, the phylogenetic tree was constructed using Neighbor-joining Method using Mega 4.0 software. Result revealed that the BMI FAR1 has a

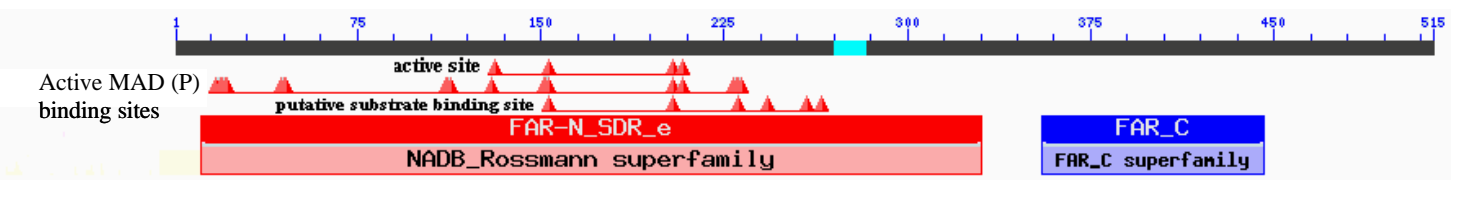

Fig. 3: The putative domains of the protein encoded by BMI FAR1

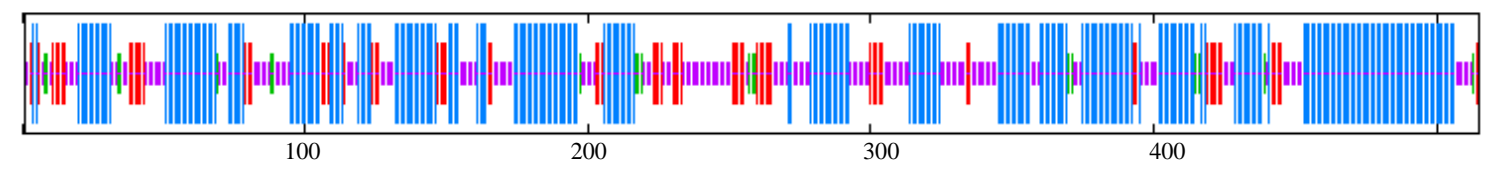

Fig. 4: The secondary structure of the BMI FAR1 protein predicted by SOPMA. Helices, strands and coils are indicated, respectively with long, middle and short vertical lines 
closer genetic relationship with the bovine, human, orangutan and monkey FAR1 than with those of horse, mouse and rat (Fig. 7).

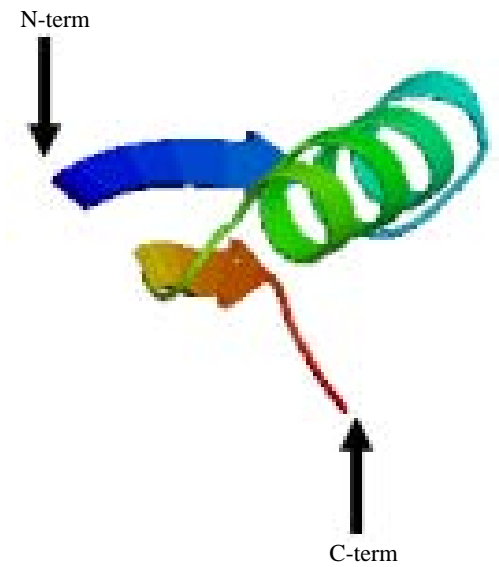

Fig. 5: The tertiary structure of BMI FAR1
mRNA tissue-specific expression profile: To check the relative expression levels of FAR1 mRNA in various porcine tissues, semi-quantitative RT-PCR was performed in $18 \mathrm{BMI}$ tissues mentioned above. The continuously expressed gene $18 \mathrm{~S}$ was used and served as an endogenous reference for determination of targeted mRNA profiles. Result revealed that BMI FAR1 gene was over-expressed in muscle, lung, ovary, skin, large intestine, spleen, small intestine and nerve fiber and almost not expressed in other 10 tissues (Fig. 8). Comparative genomics determines the relationship of genome structure and function of different species.

Researchers have learned a great deal about the function of human genes by examining their counterparts in simpler model organisms such as the mouse and some results have revealed that virtually all $(99 \%)$ of the protein-coding genes in humans align with homologues in mice and $>80 \%$ are clear 1:1 orthologs (Hardison, 2003; Liu et al., 2008). This extensive conservation in

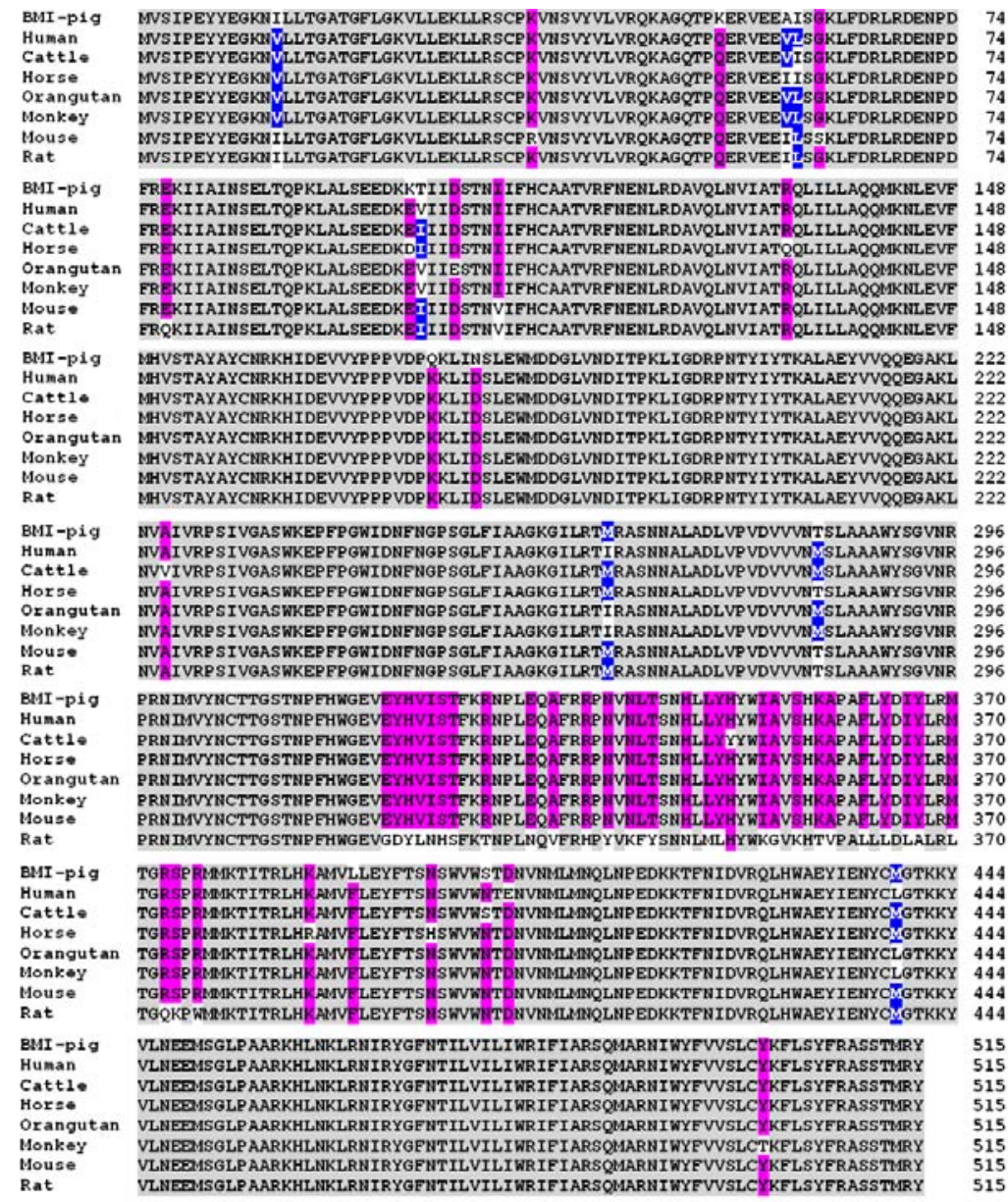

Fig. 6: Alignment of the protein encoded by the BMI FAR1 and seven other types of FAR1 from cattle (NP_001092502), horse (XP_001501396), mouse (NP_081655), orangutan (NP_001126210), human (NP_115604), monkey (XP_001093685) and rat (NP_001011933) 
protein-coding regions implied that the same proteincoding sequences may be expected in different mammals including pig. From the isolation of swine FARl gene, the researchers can find that swine FAR1 is highly homologous with FAR1 of human, bovine and other mammals. This further validated that Comparative Genomics Method is one useful tool to isolate the unknown genes, especially the conserved coding region of genes for pig. From the alignment analyses for swine FAR1 protein, researchers also find BMI FAR1 protein was not completely identity to human or other mammals. This implied that BMI FAR1 will have some differences in functions to those of human, bovine and other mammals. The phylogenetic tree analysis revealed that the BMI

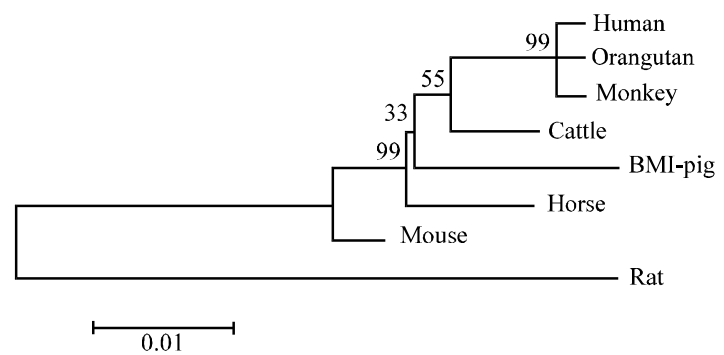

Fig. 7: Phylogenetic tree for FAR1 proteins from 8 species
FAR1 gene has a closer genetic relationship with the bovine, human, orangutan and monkey FAR1. Therefore, the researchers can use bovine, human, orangutan and monkey as model organisms to study the pig FARl gene or use pig as a model organism to study the bovine, human, orangutan and monkey FARl genes.

Most protein functions are regulated by phosphorylation/dephosphorylation, glycosylation/ deglycosylation and BMI FAR1 protein has several kinds of functional sites (such as phosphorylation sites, glycosylation sites, myristoylation sites, FAR-N_SDR_e and FAR_C protein domains) which suggest that FAR1 protein plays important functional roles through these sites and domains. MicroRNAs are small noncoding RNA. They play a role in gene expression regulation by inhibiting translation of their target mRNAs (Bartel, 2004; Zeng et al., 2003). Their target predictions showed that four Sus scrofa microRNAs (ssc-miR-542-3p, ssc-miR$22-5 p$, ssc-miR-542-3p and ssc-miR-361-3p) were found to have the corresponding target sites (209-uuuga cagauugagagaugaaa-230, 195-uuaguggcaag cuuu-209, 741 gauggauugauaacuuuaa-759 and 1438-ugugauccugauuug1452) in the BMI FAR1 coding sequence. Further
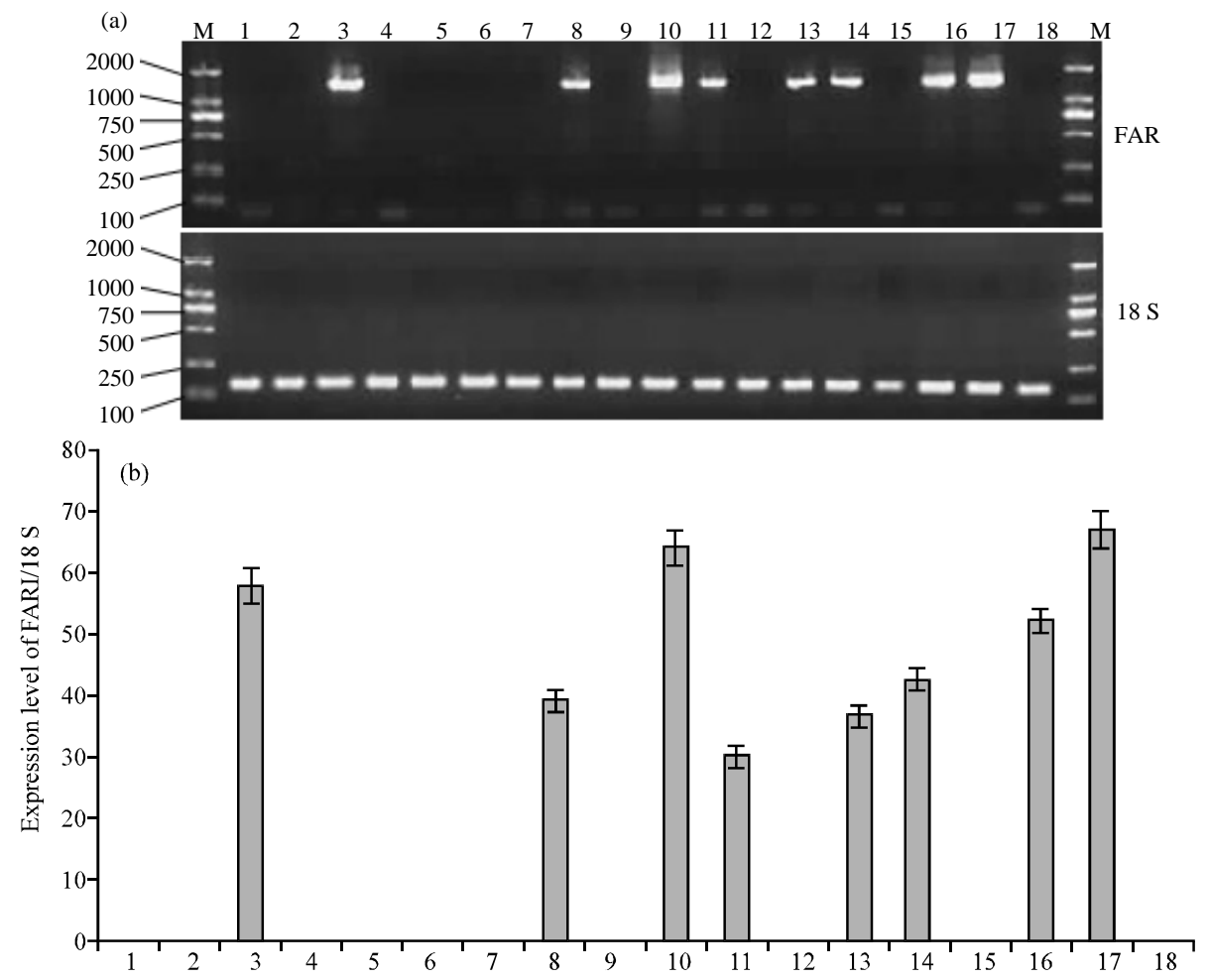

Fig. 8: Tissue expression profile of BMI FAR1 gene; 1: Lymph node; 2: Midbrain; 3: Ovary; 4: Diencephalon; 5: Cerebrum; 6: Liver; 7: Kidney; 8: Spleen; 9: Heart; 10: Lung; 11: Nerve fiber; 12: Stomach; 13: Small intestine; 14: Large intestine; 15: Pancreas; 16: Skin; 17: Muscle; 18: Fat. The 18S expression level is used for the internal control 
investigation is needed to confirm whether corresponding miRNA molecules can regulate the $F A R 1$ gene expression in swine. In this study, the researchers not only cloned the CDS sequences of the BMI FAR1 gene but also conducted the sequence analys is and tissue expression profiles analysis. From the tissue expression profile analysis, it can be seen that the gene was obviously differentially expressed in various tissues. As the researchers did not study functions at protein levels, there might be many possible reasons for differential expression of this porcine gene. The suitable explanation for this under current conditions is that the biological activities associated with the functions of the gene was demanded to a different extent in different tissues at the same time.

\section{CONCLUSION}

In this study, the researchers first isolated BMI FAR1 gene and performed necessary functional analysis and tissue expression profile analysis. Furthermore, several miRNAs were found to have the corresponding target sites in the coding sequence of BMI FAR1 by theoretical prediction. The cDNA clone, sequence information and function analysis of BMI FAR1 gene will be extremely important in elucidating the essential physiological function of FAR protein using BMI and other pigs as experimental animal models in the future.

\section{ACKNOWLEDGEMENT}

This study was supported by the National Natural Science Foundation of China (Grant No. 31160439).

\section{REFERENCES}

Bartel, D.P., 2004. MicroRNAs: Genomics, biogenesis, mechanism and function. Cell, 116: 281-297.

Blomquist, G.J., D.R. Nelson and M. de Renobales, 1987. Chemistry, biochemistry and physiology of insect cuticular lipids. Arch. Insect Biochem. Physiol., 6: 227-265.

Chen, W., X.H. Yu, K. Zhang, J. Shi, L. Schreiber, J. Shanklin and D. Zhang, 2011. Male sterile 2 encodes a plastid-localized fatty acyl ACP reductase required for pollen exine development in Arabidopsis thatiana. Plant Physiol.

Cheng, J.B. and D.W. Russell, 2004. Mammalian wax biosynthesis. I. Identification of two fatty acylcoenzyme A reductases with different substrate specificities and tissue distributions. J. Biol. Chem., 279: 37789-37797.

Crabbe, J.C., P. Metten, A.J. Cameron and D. Wahlsten, 2005. An analysis of the genetics of alcohol intoxication in inbred mice. Neurosci. Biobehav. Rev., 28: $785-802$.
Gilbert, L.I. and H. Chino, 1974. Transport of lipids in insects. J. Lipid Res., 15: 439-456.

Gilby, A.R., 1980. Transpiration, temperature and lipids in insect cuticle. Adv. Insect Physiol., 15: $1-33$.

Hardison, R.C., 2003. Comparative genomics. PLoS Biol., 1: e58-e 58 .

Liu, G. Y., S.Z. Gao, C.R. Ge and X. Zhang, 2008. Molecular characterization of the encoding regions and tissue expression analyses for three novel porcine genesHNRPA1, YIPF5 and UB2D2. Mol. Biol. Rep., 35: 519-526.

Metz, J.G., M.R. Pollard, L. Anderson, T.R. Hayes and M.W. Lassner, 2000. Purification of a jojoba embryo fatty acyl-coenzyme A reductase and expression of its cDNA in high erucic acid rapeseed. Plant Physiol., 122: 635-644.

Moto, K., T. Yoshiga, M. Yamamoto, S. Takahashi and $\mathrm{K}$. Okano et al., 2003. Pheromone gland-specific fatty-acyl reductase of the silkmoth, Bombyx mori. Proc. Natl. Acad. Sci., 100: 9156-9161.

Munn, N.J., E. Arnio, D. Liu, R.A. Zoeller and L. Liscum, 2003. Deficiency in ethanolamine plasmalogen leads to altered cholesterol transport. J. Lipid Res., 44: $182-192$

Nagan, N. and R.A. Zoeller, 2001. Plasmalogens: Biosynthesis and function. Prog. Lipid Res., 40: 199-229.

Stanley, D.W. and D.R. Nelson, 1993. Insect Lipids Chemistry, Biochemistry and Biology. University of Nebraska Press, Lincoln, ISBN: 9780803242319, Pages: 467.

Teerawanichpan, P., A.J. Robertson and X. Qui, 2010. A fatty acyl-CoA reductase highly expressed in the head of honey bee (Apis mellifera) involves biosynthesis of a wide range of aliphatic fatty alcohols. Insect Biochem. Mol. Biol., 40: 641-649.

Wanders, R.J. and H.R. Waterham, 2006. Biochemistry of mammalian peroxisomes revisited. Annu. Rev. Biochem., 75: 295-332.

Yu, P., L. Zhang, S. Li, Y. Li and J. Cheng et al., 2004. Screening and analysis of porcine endogenous retrovirus in Chinese banna minipig inbred line. Transplant. Proc., 36: 2485-2487.

Zeng, R. and Y.Z. Zeng, 2005. Molecular cloning and characterization of SLA-DR genes in the 133-family of the banna mini-pig inbred line. Anim. Genet, 36: 267-269.

Zeng, Y., R. Yi, and B.R. Cullen, 2003. MicroRNAs and small interfering RNAs can inhibit mRNA expression by similar mechanisms. Proc. Natl. Acad. Sci., 100: 9779-9784. 